\title{
Removal of a malpositioned implant and immediate re- implantation during healing period
}

\author{
${\text { Dimitris Nikolidakis }{ }^{1 *} \text { and Nikitas Sykaras }}^{2}$ \\ ${ }^{1}$ Private Periodontal Practice, Heraklion, Greece \\ ${ }^{2}$ Department of Prosthodontics, Dental school, University of Athens, Greece
}

\section{Introduction}

Dental implants provide a strong foundation for fixed or removable prosthesis that are made to replace natural teeth. Nevertheless, the restoration of teeth with implant supported crowns or bridges, is a technique-sensitive procedure and can be a challenge for the surgicalrestorative team [1].

Several factors such as the quantity and quality of available alveolar residual ridge that, the correct positioning of the implant, the soft tissue biotype, the selection of the implant abutment, the provisional restoration and the fabrication of the definitive crown affect clinical outcomes [2].

The successful placement of dental implants in the edentulous upper or lower jaw can be compromised by the lack of adequate alveolar bone volume and the amount of available bone is one of the critical factors determining the ultimate success of the implant [3].

Implant angulation is yet another determinant for implant success. Proper angulation should be determined according to the future prosthesis with the consideration of bucco-lingual, apico-coronal, and mesio-distal positions.

Coronal positioning of the implant is required to mask the metallic view of the implant while, placing the implant-abutment surface more apically also leads to clinical difficulties regarding the establishment of the biologic width around the implant [4].

Also, the implant should be angulated correctly in the buccolingual and mesio-distal planes for optimum function and esthetics. Endosseous implants distribute occlusal loads most effectively when forces are applied in an axial direction. As implant angulation approaches or exceeds 25 degrees, the supporting bone is severely compromised through transmission of occlusal forces. Moreover, if an implant is inclined and the prosthetic reconstruction is offset relative to the implant head, the inclination will results in poor esthetic outcomes as well as long-term biomechanical instability [5].

To maximize the outcome of implant placement, the use of advanced radiographic procedures such as computerized tomography, along with fabrication of surgical guides, has been advocated to inform surgeons of ideal implant location. More recently, simulation computer software has been introduced to view radiographic images and test potential implant locations and yet, surgical guides are processed based on ideal tooth position [6].

However, recent technology have allowed more predictable placement of dental implants, the malposition of endosseous implants is still not uncommon. Therefore, several techniques have been described to surgically or prosthodontically compensate for improperly inclined implants [7-10].

Additionally, the removal of endosseous implants may be performed in cases of severe implant malposition and in patients with serious complaints despite that the explantation procedure can be complicated in some situations [11].

The present clinical case report describes an alternative technique for the surgical management of an unfavorable implant position by relocating the same implant during the postoperative period.

\section{Case report}

\section{Patient intake and diagnosis}

A 39-year-old Caucasian non-smoking female was referred to a specialist for implant treatment. The patient was in good general health and did not have any subjective dental complaints except of a malpositioned dental implant. The dental history revealed that, six months ago, her general dentist extracted the first left lower molar (tooth no 19) planning to replace it with a dental implant. After completion of a five months postoperative period, the dentist indented to place an implant with diameter $4.8 \mathrm{~mm}$ and length of $12 \mathrm{~mm}$ (Straumann ${ }^{\circ}$, Standard Plus Implant System, Institute Straumann AG, Basel, Switzerland). During surgery, he noticed that the alveolar socket of the extracted tooth (tooth no 19) was not fully healed despite the prolonged postoperative period (Figure 1) and in agreement with the patient, decided to insert the implant in the position of the distal root (distal aspect of the socket) and close to second molar (Figure 2). After suture removal, he reconsidered the treatment plan based also on his dental technician opinion and patient will, as a successful prosthetic rehabilitation couldn't be ensured (Figure 3).

Therefore, he referred the patient for implant removal and re implantation.

\section{Treatment planning}

During the oral examination and evaluation of the periodontal status, the oral hygiene appeared to be adequate and no further clinical findings were present. Based on the dental history, the clinical and

Correspondence to: Dimitris Nikolidakis, DDS, MSc, $\mathrm{PhD}$, Private periodontal practice, Heraklion, Greece, Tel: (0030) 2810288611; Fax: (0030) 2810288611 Email: d.nikolidakis@gmail.com

Received: November 21, 2017; Accepted: December 05, 2017; Published: December 08, 2017 


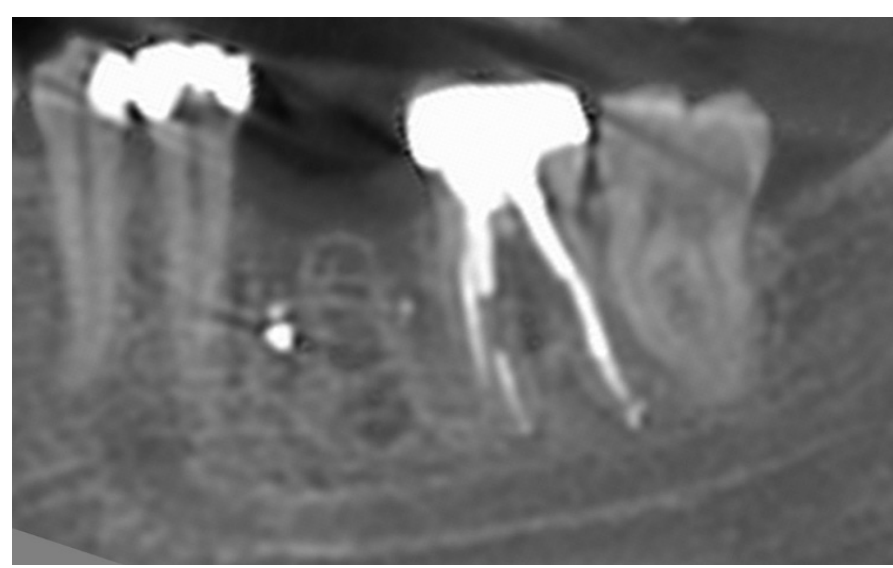

Figure 1. After completion of a five months postextraction period, the alveolar socket of the extracted tooth (no 19) was not fully healed.

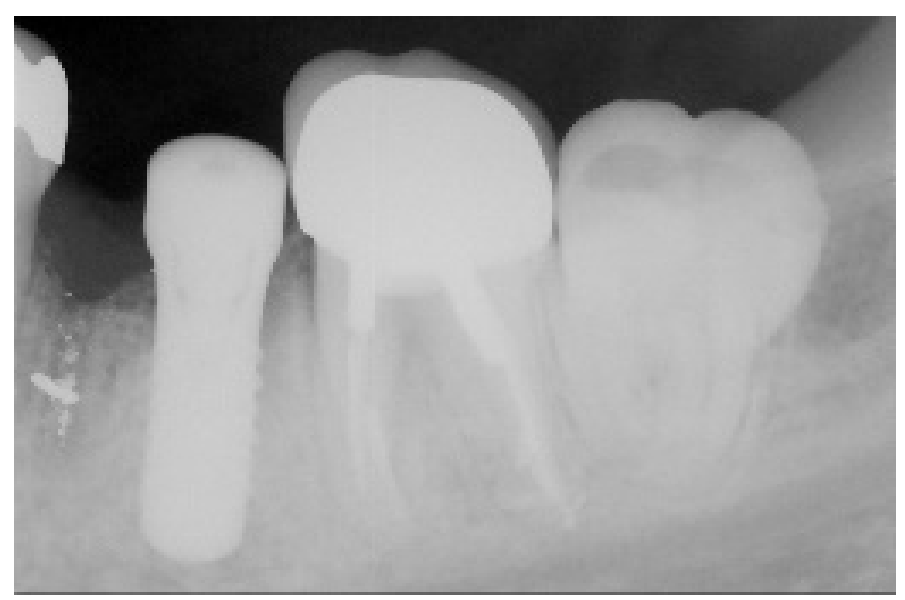

Figure 2. An implant was placed in the position of the distal root of the extracted first lower molar and close to second molar.

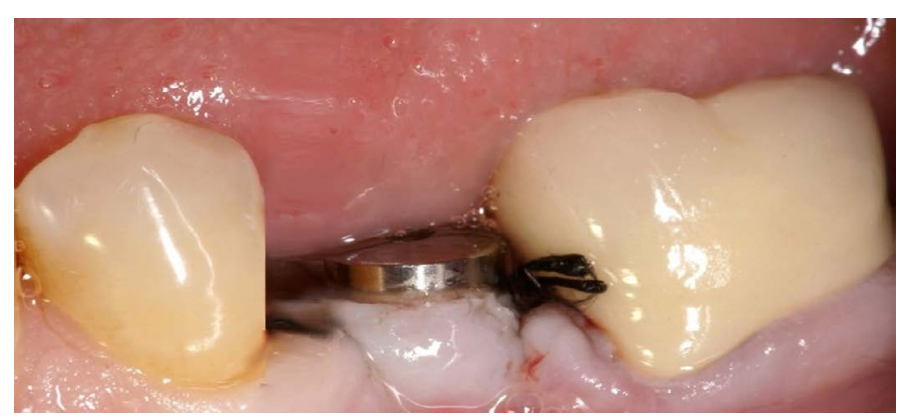

Figure 3. Due to implant malposition a successful prosthetic rehabilitation couldn't be ensured.

radiographic examinations, the treatment plan included atraumatic removal of the implant and immediate relocation of the same implant in a prosthetically suitable position. The surgical procedure was scheduled in a period of four to six weeks after first insertion because this timepoint combined an adequate soft tissue healing after initial implant placing and avoidance of osseointegration that could compromise atraumatic removal.

\section{Surgical procedure}

Preoperatively, the alveolar bone volume was assessed based on previous CT scan radiographic examination (Figure 4). Adequate bone height of $15 \mathrm{~mm}$ away from alveolar nerve and width of $9 \mathrm{~mm}$ was estimated. The relocation of the same implant $(4.8 \times 12 \mathrm{~mm})$ in the middle of the edentulous area was attainable. The patient received antibiotic prophylaxis prior to surgery.

After careful elevation of the mucosal layer, the alveolar ridge between lower left second premolar and molar was exposed and the implant was removed without any damage of the alveolar bone or the material (Figure 5). Implant removal was accomplished by applying the manufacturer implant transfer part and the ratchet and turning it initially clockwise (like ending insertion procedure) with a high torque more than $50 \mathrm{~N} / \mathrm{cm}$ up to disconnect the immature bone implant contact and afterwards counterclockwise for implant retrieval. The removed implant was submerged in a dish with blood and saline and used again after socket debridement (Figure 6). Thereafter, the alveolar socket (mesial aspect) was exposed and cleaned with bone curettes as complete wound closure had not been achieved. The removal of debris from the socket was completed by irrigation with saline. The empty space of the mesial socket defect and the implant cavity after explantation was filled with $0.5 \mathrm{cc}$ xenograft granules of 0.25 to $1 \mathrm{~mm}$ in diameter (BioOss ${ }^{\circ}$, Geistlich Pharma AG, Wolhusen, Switzerland) to facilitate the proper guidance of drilling and implant insertion. Therefore, implant placement was performed in a proper position according to manufacturer guidelines with the exception of low speed drilling (200-400 rpm) with minimal water cooling to avoid graft particle loss (Figure 7). The implant remained submerged with flap closure. Sutures were applied and postoperative instructions were given. Antibiotic coverage of $500 \mathrm{mg}$ amoxicilline times per day was applied. Postsurgical pain and edema were controlled with 600 mg ibuprofen three times per day, and the patient was instructed to rinse twice daily with $0.12 \%$ chlorhexidine for two weeks and to use modified oral hygiene procedures in the treated area for the first six postoperative weeks.

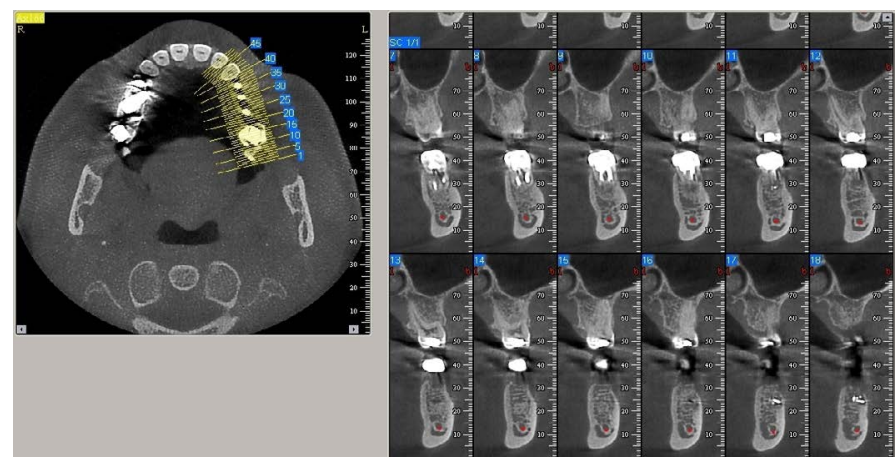

Figure 4. Preoperatively, the alveolar bone volume was assessed based on previous CT scan radiographic examination.

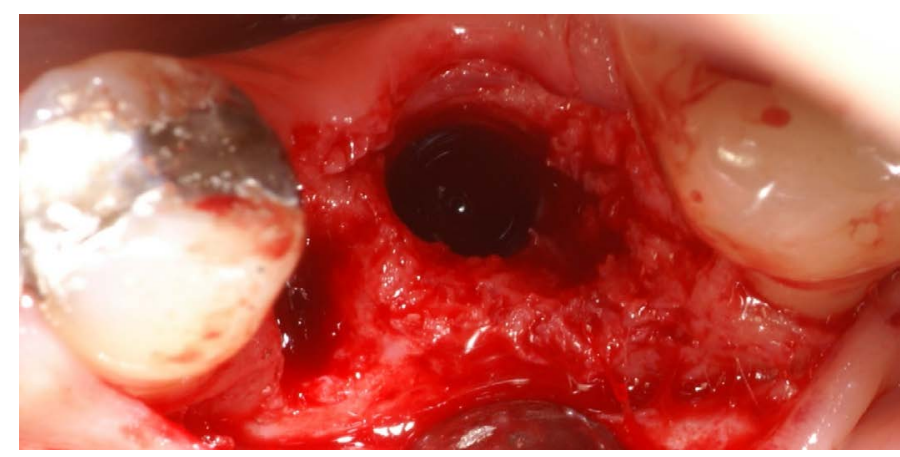

Figure 5. After careful elevation of the mucosal layer, the alveolar ridge between lower left second premolar and molar was exposed and the implant was removed. 


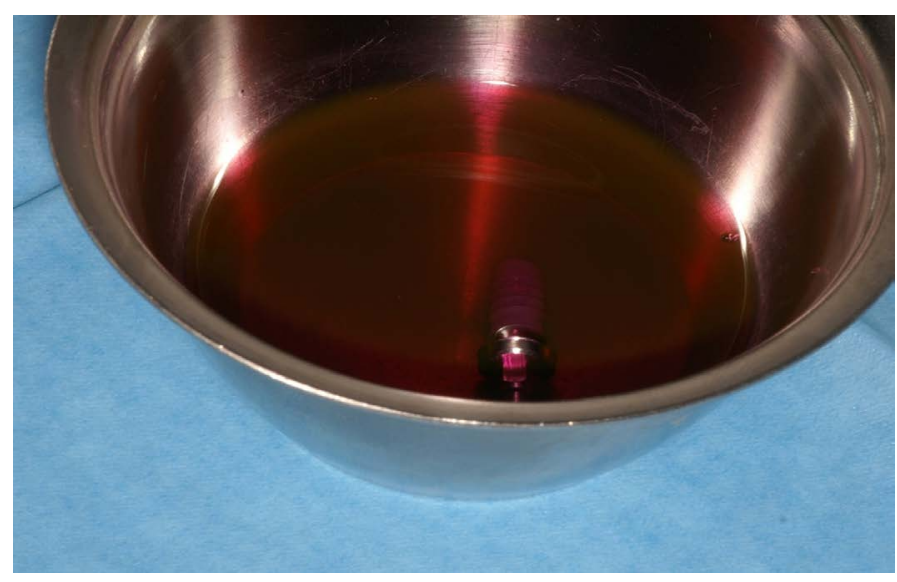

Figure 6. The removed implant was submerged in a dish with blood and saline and used again after socket debridement.

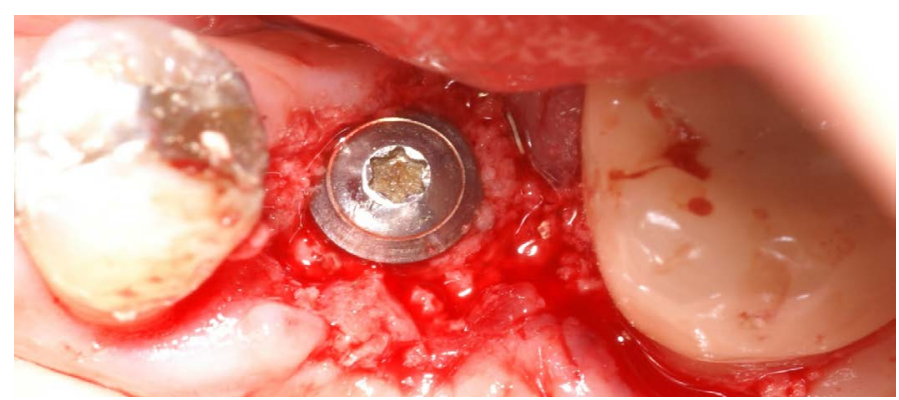

Figure 7. Implant placement was performed according to manufacturer surgical guidelines in a proper position.

\section{Follow-up care}

The patient was examined two weeks later and the sutures were removed. Radiographic examination was also performed (Figure 8). The patient was maintained in a supportive care program and control appointments once per month were scheduled. Five months after insertion, the implant was exposed and an abutment was connected. A single metal ceramic crown was fabricated four weeks later (Figure 9). One year after prosthetic rehabilitation, a reevaluation of the case was performed. The general periodontal status was stable and the patient had achieved a very good level of plaque control. No dental complaints were reported. Clinical examination of the implant placed in the augmented area revealed no mobility and healthy peri-implant mucosa. The radiographic examination confirmed bone-implant contact without any sign of radiolucency around the implant (Figure 10). Further the patient has completed three years of functional loading, following annual check-up with no additional clinical findings.

\section{Discussion}

An important determinant for implant success is implant angulation and position and despite recent technology have allowed more predictable placement of dental implants, the malposition of endosseous implants is still a nightmare for surgeons and prosthodontists. Therefore, numerous techniques have been described for malpositioned endosseous implants to surgically or prosthodontically correct the improper inclination.

From the prosthetic aspect, unacceptably inclined implants can lead to more technically demanding prosthodontic management to compensate for the potential esthetic complication. Measures like long time provilization to allow the establishment of soft-tissue contour and optimize gingival esthetics, placing of implant shoulder deep enough below the cemento-enamel junction of the adjacent teeth to contribute to a more favorable emergence profile or using custom abutment as it can be easily shaped to the desired definitive contour may enhance the overall esthetic outcome [12,13].

Regarding the surgical point of view, to prevent excessive angulation, the surgeon should evaluate the position of the osteotomy after use of the pilot drill by placing a parallel pin in the pilot hole and taking a radiograph. If the angulation is not satisfactory, a drill can be used to adjust the angulation before continuing preparation of the implant site. However, in case of unacceptably inclined implants, few reports proposed repositioning of the prosthetically unfavorable implant by piezoelectric osteotomy or vertical distraction osteogenesis $[7,10]$.

Additionally, removal of endosseous implants may be performed in cases of severe implant malposition. However, bone and soft tissue defects may exist following explantation and guided bone regeneration in conjunction with soft tissue grafting are used to attempt correction of the defect although a difficulty in accomplishing complete correction of the deficiencies has been described [14].

Based on these limitations, numerous companies propose extraction kits that allow dentists to retreat cases by extracting osseointegrated implants with an atraumatic manner using a set of particular trephines to avoid high forces and improve the final results, achieving the best outcomes for patients [15].

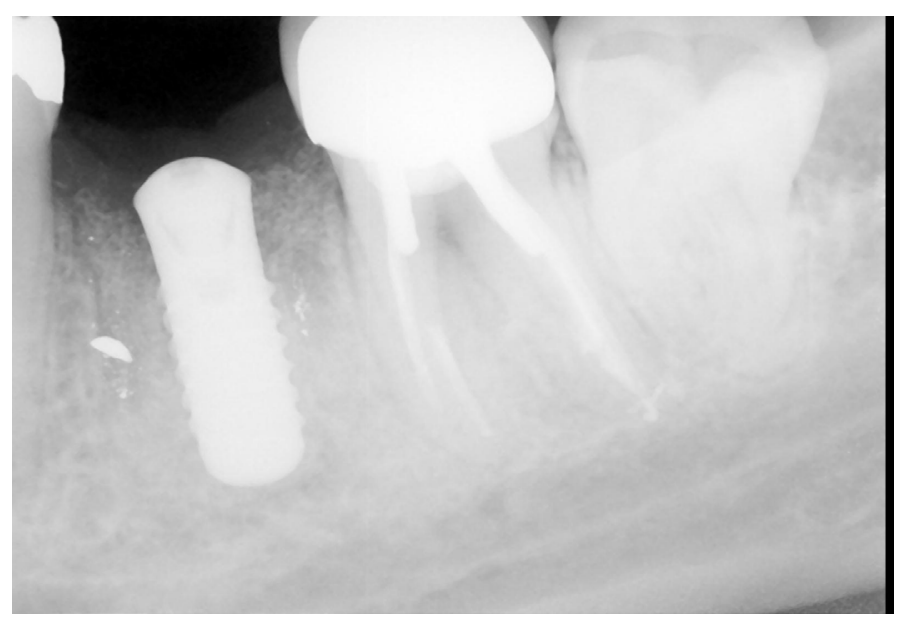

Figure 8. Radiographic examination was performed two weeks after implant relocation in a suitable position.

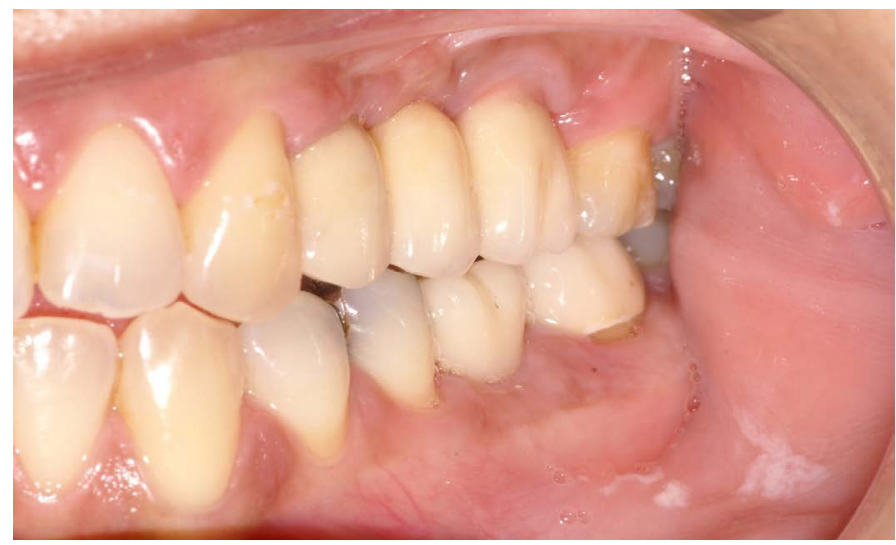

Figure 9. Clinical view of the prosthesis that was fabricated six months after implant placement. 


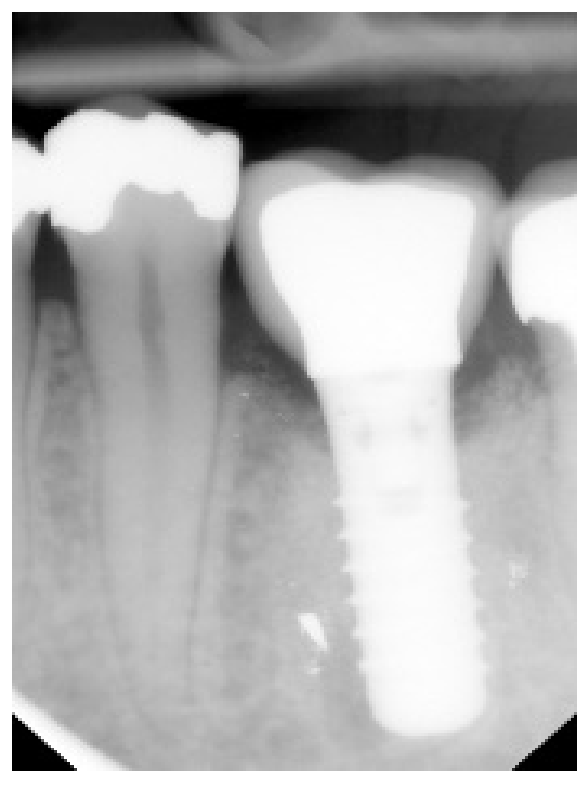

Figure 10. The radiographic examination confirmed bone-implant contact without any sign of radiolucency around the implant.

Despite the survival rate of dental implants that were placed in sites where failed implants were previously removed is very high [16], previous implant removal may discourage practitioners to perform implant placement in the same position or in the same session.

However, there is lack of evidence regarding the atraumatic explantation of malpositioned implant that could be placed the same itself again in a suitable position.

Finally, in order to avoid the retrieval of a malpositioned dental implant, the importance of thorough communication among all members of the dental implant team should be emphasized.

\section{Summary}

Correct positioning of the implant in a three-dimensional direction is an important key factor that contributes to the success of the restoration and tooth replacement. This clinical case report describes an alternative technique for the surgical management of an unfavorable implant position by relocating the same implant during the postoperative period. The malpositioned implant was removed without any damage of the alveolar bone or the material and placed again in a suitable position. No adverse reactions were observed. Thereafter, the patient has completed three years of functional loading, following annual check-up with no complains or clinical problems.

\section{Disclosure}

The authors have no financial interest in any of the companies mentioned in this article and received no compensation for writing this article.

\section{References}

1. Sadan A, Blatz MB, Salinas TJ, Block MS (2004) Single-implant restorations: a contemporary approach for achieving a predictable outcome. J Oral Maxillofac Surg 62: 73-81. [Crossref]

2. Buser D, Martin W, Belser UC (2004) Optimizing esthetics for implant restorations in the anterior maxilla: Anatomic and surgical considerations. Int J Oral Maxillofac Implants 19: 43-61. [Crossref]

3. Belser UC, Buser D, Hess D, Schmid B, Bernard JP, Lang NP (1998) Aesthetic implant restorations in partially edentulous patients - A critical appraisal. Periodontol 2000 17: 132-150. [Crossref]

4. Hermann JS, Buser D, Schenk RK, Higginbottom FL, Cochran DL (2000) Biologic width around titanium implants. A physiologically formed and stable dimension over time. Clin Oral Implants Res 11: 1-11. [Crossref]

5. Misch K, Wang HL (2008) Implant surgery complications: etiology and treatment. Implant Dent 17: 159-168. [Crossref]

6. Jabero M, Sarment DP (2006) Advanced surgical guidance technology: a review. Implant Dent 15: 135-142. [Crossref]

7. Stacchi C, Costantinides F, Biasotto M, Di Lenarda R (2008) Relocation of malpositioned maxillary implant with piezoelectric osteotomies: a case report. Int $J$ Periodontics Restorative Dent 28: 489-495. [Crossref]

8. Lewis S, Avera S, Engleman M, Beumer J 3rd (1989) The restoration of improperly inclined osseointegrated implants. Int J Oral Maxillofac Implants 4: 147-152. [Crossref]

9. Duff RE, Razzoog ME (2006) Management of a partially edentulous patient with malpositioned implants, using all-ceramic abutments and all-ceramic restorations: A clinical report. J Prosthet Dent 96: 309-312.

10. Oduncuoglu BF, Alaaddinoglu EE, Oguz Y, et al. (2011) Repositioning a prosthetically unfavorable implant by vertical distraction osteogenesis. J Oral Maxillofac Surg 69 : $1628-1632$.

11. ten Bruggenkate CM, Sutter F, van den Berg JP, Oosterbeek HS (1994) Explanation procedure with special emphasis on the ITI implant system. Int J Oral Maxillofac Implants 9: 223-229. [Crossref]

12. Jemt $\mathrm{T}$ (1999) Restoring the gingival contour by means of provisional resin crowns after single-implant treatment. Int J Periodontics Restorative Dent 19: 20-29. [Crossref]

13. Tan PL, Dunne JT Jr (2004) An esthetic comparison of a metal ceramic crown and cast metal abutment with an all-ceramic crown and zirconia abutment: a clinical report. $J$ Prosthet Dent 91: 215-218. [Crossref]

14. Horvath SD, Kohal RJ (2011) Rehabilitation of an extensive anterior explanation defect--a case report. Quintessence Int 42: 539-545.

15. Anitua E, Orive G (2012) A new approach for atraumatic implant explantation and immediate implant installation. Oral Surg Oral Med Oral Pathol Oral Radiol 113: e19-25. [Crossref]

16. Mardinger O, Ben Zvi Y, Chaushu G, Nissan J, Manor Y (2012) A retrospective analysis of replacing dental implants in previously failed sites. Oral Surg Oral Med Oral Pathol Oral Radiol 114: 290-293. [Crossref]

Copyright: $@ 2017$ Nikolidakis D. This is an open-access article distributed under the terms of the Creative Commons Attribution License, which permits unrestricted use, distribution, and reproduction in any medium, provided the original author and source are credited. 